\title{
Effects of core needle biopsy and subsequent neoadjuvant chemotherapy on molecular alterations and outcome in breast cancer
}

This article was published in the following Dove Press journal:

OncoTargets and Therapy

\author{
Lingmin $\mathrm{Xie}^{1}$ \\ Xiaolei $\mathrm{Li}^{2}$ \\ Qinchuan Wang' \\ Jichun Zhou' \\ Jun Shen' \\ Lixi Luo' \\ Yi Lu' \\ Linbo Wang'
}

'Division of Surgical Oncology, Sir Run Run Shaw Hospital, Zhejiang University School of Medicine,

Zhejiang, ${ }^{2}$ Division of Surgical

Oncology, The First People's Hospital of Wenling, Zhejiang, China
Correspondence: Linbo Wang Division of Surgical Oncology, Sir Run Run Shaw Hospital, Zhejiang University School of Medicine, 3 Qingchun Road, Hangzhou City, Zhejiang 310016,

Zhejiang, China

Tel +86057l86002176

Email linbowang@zju.edu.cn
Objectives: The aim of our study is to evaluate the effect of core needle biopsy (CNB) and subsequent neoadjuvant chemotherapy (NAC) on the expression of estrogen receptor (ER), progesterone receptor (PR), human epidermal growth hormone receptor 2 (HER2) and Ki67 in breast cancer, and the associated influencing factors.

Materials and methods: In this retrospective cohort study, 143 patients with primary operable breast cancer who received NAC were included. ER, PR, HER2 and Ki67 statuses were compared between pretreatment and posttreatment residual samples. A control group of paired core and excision tumors from 123 patients who did not receive NAC within the same study period was also assessed. Data on patients' clinicopathologic features were collected to identify associated influencing factors.

Results: Ki67 value significantly increased in excision tumors compared with paired core samples in controls without presurgery treatment $(P<0.01)$, which was associated with the pathologic lymph node status and the interaction of PR and HER2 status ( $P=0.008$ and 0.028 , respectively). In 143 patients who underwent NAC, a significant decrease was observed in the expression of PR and Ki67 after NAC ( $P=0.003$ and $P<0.01$, respectively). Further subgroup analysis showed that $\mathrm{PR}$ decrease was more obvious in premenopausal patients and Luminal A patients ( $P=0.006$ and 0.002 , respectively).

Conclusion: Core samples could provide more reliable information on determination of molecular subtype than surgical excisions. Decreases in PR and Ki67 expression following NAC could be used as positive prognostic factors. We recommend repeat testing of these biologic markers following NAC for the sake of better disease management. To the best of our knowledge, this is the most comprehensive study to analyze the effect of neoadjuvant chemotherapy on molecular alteration and its associated influencing factors after reporting a CNB-associated Ki67 increase in the same study.

Keywords: breast cancer, molecular change, neoadjuvant chemotherapy, core needle biopsy

\section{Introduction}

Neoadjuvant chemotherapy (NAC) has been widely used in locally advanced breast cancer with the aim of downstaging and facilitating conservative surgery. ${ }^{1}$ It also gives individual evaluation of responses to chemotherapy and provides prognostic information to guide future management strategies., ${ }^{2,3}$ Several studies have reported that the extent of residual disease, including the primary tumor (size, cellularity, and in situ disease) and the involved lymph nodes (number and size), is an independent predictor of disease-free survival (DFS) and overall survival. ${ }^{4-6}$

Testing the tumor core biopsy samples for estrogen receptor (ER), progesterone receptor (PR), human epidermal growth factor receptor 2 (HER2) and Ki67 is a 
prerequisite for selecting patients into the neoadjuvant route and deciding on drug combinations. Previous studies have reported a change in the expression of ER, PR, HER2 and Ki67 following NAC, and have suggested this alteration to be a potential prognostic factor. ${ }^{7,8}$ The post-neoadjuvant treatment biomarker statues were integrated in a model for outcome prediction. ${ }^{9-12}$

Although the effect of NAC on biomarker expression has been studied, the results were controversial. First, most of these studies made a comparison between pretreatment and posttreatment residual samples from patients who underwent NAC in a self-control method, overlooking variations in the biomarker determining process. Tissue processing and sample fixation in immunohistochemical procedures can reportedly cause inconsistent results of biomarker assessment. Second, intra- and interobserver variability can also make a contribution. Third, breast cancer is generally known as a heterogeneous disease with intratumoral heterogeneity, making the initial core needle biopsy (CNB) not the representative of the whole tumor. ${ }^{13,14}$

In addition, $\mathrm{CNB}$ itself might induce biomarker change. Several studies have shown a higher level of Ki67 in surgical tumor excisions than in core samples. ${ }^{15,16}$ The study by Chen et al found that Ki67 value significantly increased after $\mathrm{CNB}$, and this was associated with the surgery time interval (STI) and molecular subtype. ${ }^{16}$ This result is supported by Kim et al, who further demonstrated that a substantial discordance in Ki67 after biopsy was significantly associated with variables including tumor size $>1 \mathrm{~cm}$, negative PR expression, grade III cancer and age 35 years. ${ }^{17}$

Thus, interfered by the above factors, results reported in previous self-control studies might be the collective effect of CNB and NAC. In order to identify the genuine effect of NAC on biomarkers, CNB and analytical factors have to be taken into consideration. The aim of our study is to evaluate the biomarker alterations that occur after CNB and subsequent NAC in a more precise way and to explore the associated risk factors. Surgical excision samples (SRSs) and paired core samples both from patients treated with NAC and patients without presurgery systemic therapy were included. Biomarker change was assessed through intergroup comparison, and associated influencing factors on biomarker change were collected and analyzed.

\section{Materials and methods Case selection}

Included in this study were patients with primary operable breast carcinomas diagnosed from 2005 to 2015 at Sir Run Run Shaw Hospital affiliated to Zhejiang University School of Medicine through retrospective review of surgical pathology report databases and medical chart review.

The enrollment criteria of NAC group were as follows: 1) female; 2) confirmed diagnosis of breast carcinoma by CNB; 3) solitary lesion; 4) no previous chemotherapy, endocrine therapy, radiotherapy or target therapy and 5) no polymerase chain reaction. after NAC. Of 188 patients who underwent NAC for primary and operable invasive carcinoma, $23.9 \%$ of patients (45 cases) achieved pathologic complete response and were excluded due to the lack of residual tumors. The remaining 143 subjects with residual disease were allocated to the NAC group. The control group consisted of 123 patients who did not undergo NAC within the same study period. Consecutive core biopsies and tumor slides from the surgical excision were identified and stained for ER, PR, HER2 and Ki67.

Data on medical history and patient characteristics (including age, menopausal status, tumor size, lymph node status, hormone receptor [HR], HER2, Ki67 status at diagnosis and surgery, tumor phenotype, NAC regimens and cycles, response to NAC, STI between CNB and surgery) were collected. Effects of NAC on breast cancer phenotype, ER/PR and HER2 expression, axillary lymph node status and tumor size were obtained from the pathologic specimens at surgery in the control group and from clinical assessment at diagnosis in the NAC group. Tumor size in the NAC group was presented as the maximum diameter of the main tumor mass under ultrasound, which led to assessment of missing data of 10 patients by magnetic resonance imaging. The clinicopathologic features of all patients at biopsy are summarized in Table 1.

\section{Treatment}

In the NAC group, patients received NAC regimens consisting of docetaxel, epirubicin and cyclophosphamide (TEC); 5-fluorouracil, epirubicin and cyclophosphamide (FEC); epirubicin and cyclophosphamide (EC) or other agents every 3 weeks. Median NAC cycle (range, 1-8 cycles) was 3 cycles. Four patients with sequential administration of taxanes received more than 8 cycles preoperatively before chemotherapy. In the control group, patients underwent surgery directly following diagnostic CNB. CNB and surgery dates were retrieved to calculate the STI after CNB.

\section{Immunohistochemistry}

CNB and SRS samples were fixed in 10\% neutral buffered formalin within $30 \mathrm{~min}$ after tumor removal, and the fixation interval was 24 hours. Immunohistochemistry (IHC) streptavidin-peroxidase (S-P) method was used to detect 
Table I Clinicopathologic features of patients at biopsy $(\mathrm{N}=266)$

\begin{tabular}{|c|c|c|c|}
\hline Clinical variables & Level & $\begin{array}{l}\text { Number in } \\
\text { control group }\end{array}$ & $\begin{array}{l}\text { Number in } \\
\text { NAC group }\end{array}$ \\
\hline \multirow[t]{4}{*}{ Age at diagnosis } & $<35$ & 2 & 10 \\
\hline & $35-50$ & 47 & 66 \\
\hline & $51-65$ & 56 & 59 \\
\hline & $>65$ & 18 & 8 \\
\hline \multirow[t]{3}{*}{ Menopausal status } & Premenopausal & 45 & 71 \\
\hline & Postmenopausal & 74 & 66 \\
\hline & Unknown & 4 & 6 \\
\hline \multirow[t]{4}{*}{ Tumor size $(\mathrm{cm})^{\mathrm{a}}$} & $\leq 2$ & 47 & 27 \\
\hline & $2-5$ & 66 & 95 \\
\hline & $>5$ & 10 & II \\
\hline & Unknown & 0 & 10 \\
\hline \multirow[t]{3}{*}{ Axillary lymph node } & Negative & 66 & 53 \\
\hline & Positive & 55 & 90 \\
\hline & Unknown & 2 & 0 \\
\hline \multirow[t]{5}{*}{$\mathrm{ER}^{\mathrm{b}}$} & 0 & 33 & 76 \\
\hline & 1 & 9 & 22 \\
\hline & 2 & 23 & 13 \\
\hline & 3 & 48 & 29 \\
\hline & Unknown & 0 & 3 \\
\hline \multirow[t]{5}{*}{$\mathrm{PR}^{\mathrm{b}}$} & 0 & 50 & 76 \\
\hline & I & 21 & 22 \\
\hline & 2 & 22 & 13 \\
\hline & 3 & 30 & 29 \\
\hline & Unknown & 0 & 3 \\
\hline \multirow[t]{3}{*}{ Ki67 (\%) } & $<14$ & 55 & 20 \\
\hline & $\geq 14$ & 68 & 70 \\
\hline & Unknown & 0 & 53 \\
\hline \multirow{3}{*}{ HER2 status ${ }^{b}$} & Negative & 98 & 102 \\
\hline & Positive & 25 & 40 \\
\hline & Unknown & 0 & 1 \\
\hline \multirow[t]{5}{*}{ Molecular subtype ${ }^{b}$} & Luminal A & 33 & 14 \\
\hline & Luminal B & 61 & 52 \\
\hline & HER2 positive & 14 & 27 \\
\hline & Triple negative & 15 & 29 \\
\hline & Unknown & 0 & 21 \\
\hline \multirow[t]{4}{*}{ NAC regimen } & FAC or FEC & - & 57 \\
\hline & TEC & - & 51 \\
\hline & $\mathrm{AC}$ or $\mathrm{EC}$ & - & 13 \\
\hline & Others & - & 22 \\
\hline \multirow[t]{5}{*}{ NAC cycles } & $\mathrm{I}-2$ & - & 27 \\
\hline & $3-4$ & - & 76 \\
\hline & $5-6$ & - & 27 \\
\hline & $7-8$ & - & 9 \\
\hline & $>8$ & - & 4 \\
\hline \multirow{3}{*}{$\begin{array}{l}\text { Surgery time interval } \\
\text { (days) }\end{array}$} & $0-2$ & 23 & - \\
\hline & $3-4$ & 34 & - \\
\hline & $\geq 5$ & 66 & - \\
\hline
\end{tabular}

Notes: axillary lymph node status and tumor size were obtained from pathologic specimen at surgery in the control group and from ultrasound assessment at diagnosis in the NAC group. 'Expression status in CNB sample at diagnosis.

Abbreviations: AC, Adriamycin and cyclophosphamide; EC, epirubicin and cyclophosphamide; CNB, core needle biopsy; ER, estrogen receptor; FEC, 5-fluorouracil, epirubicin and cyclophosphamide; HER2, human epidermal growth hormone receptor 2; NAC, neoadjuvant chemotherapy; PR, progesterone receptor; TEC, docetaxel, epirubicin and cyclophosphamide.

the expression of biomarkers in this study, all of which were performed by the Department of Pathology, Sir Run Run Shaw Hospital, Zhejiang University School of Medicine. All IHC section observations were scored independently under microscopy by two pathologists blinded to the clinical information of the subjects. The data were presented as average values of the observation of the two pathologists.

Tumors with $>10 \%$ positive invasive cell nuclear staining were classified as ER+ or PR+, whereas tumors with $<10 \%$ staining were regarded as negative. $\mathrm{ER}+$ or $\mathrm{PR}+$ tumor was further scored as follows: + score, $10 \%-25 \%$ staining of tumor cells; $2+, 26 \%-50 \%$ staining of tumor cells and $3+,>50 \%$ staining of tumor cells. For Ki67 expression measurement, 2,000 cells were equally counted in both hotspot and negative areas in slice. Ki67 expression was assessed as the percentage of positive invasive tumor cells with any nuclear staining and recorded as the mean percentage of positive cells. The Ki67 cutoff value in determining Luminal status was 14\%. The 2007 American Society of Clinical Oncology/College of American Pathologists guidelines were applied in the HER2 status evaluation. For the determination of HER2 protein overexpression, a 0 score is defined as tumors with no staining; a $1+$ score as weak, incomplete membrane staining in any proportion of tumor cells; a 2+ score as complete membrane staining that is either nonuniform or weak in intensity, but with obvious circumferential distribution in at least $10 \%$ of cells or intense, complete membrane staining of $<30 \%$ tumor cells; and a $3+$ score as uniform intense membrane staining of $>30 \%$ of invasive tumor cells. Due to the absence of fluorescence in situ hybridization assessment, +++ score was defined as HER2 positive (HER2 overexpression), whereas the rest was HER2 negative. Biomarker IHC pictures are shown in Figure 1. ER/PR/HER2 status and Ki67 value were obtained from the original pathology reports. There were four breast cancer subtypes classified according to the 2011 St Gallen breast cancer consensus: Luminal A (HR+/HER2-, Ki67 < 14\%), Luminal B (HR+/ HER2-, Ki67 $>14 \%$ or HR+/HER2+), triple negative (HR-/ HER2-) and HER2 positive (HR-/HER2+). ${ }^{18}$ The change in biomarkers after surgery or NAC was presented as the alteration of scores $(-,+,++,+++)$ for ER, PR and HER2, and the percentage values for Ki67. In this study, biomarker change was calculated as postoperative biomarker levels minus preoperative biomarker levels.

For patients in the control group, ER, PR, Ki67 and HER2 IHC profiles were available for both pretreatment CNB and posttreatment segmental or total mastectomy specimens. In the NAC group, however, a part of biomarker data was missing. Paired-Ki67 value in 57 patients (53 patients' pretreatment data and 47 patients' posttreatment data), paired-ER and PR expressions in 5 patients ( 3 patients' pretreatment data and 2 patients' posttreatment data) and HER 2 status in 1 patient before NAC were not available. 

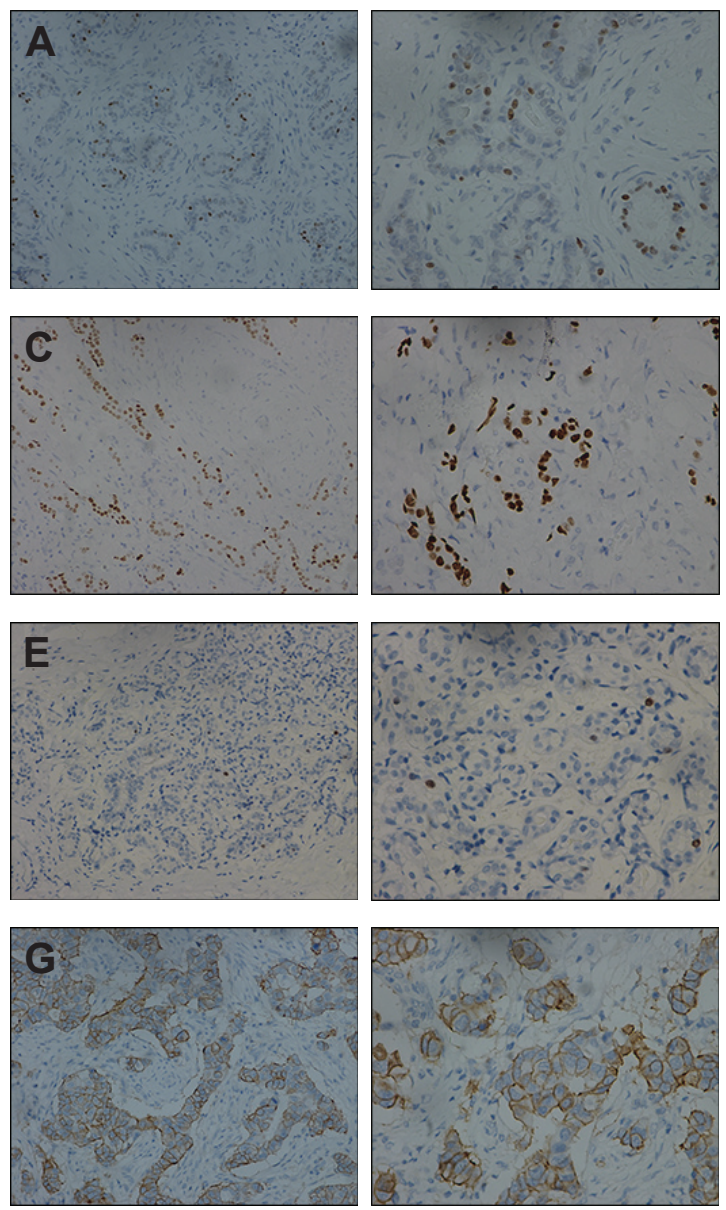
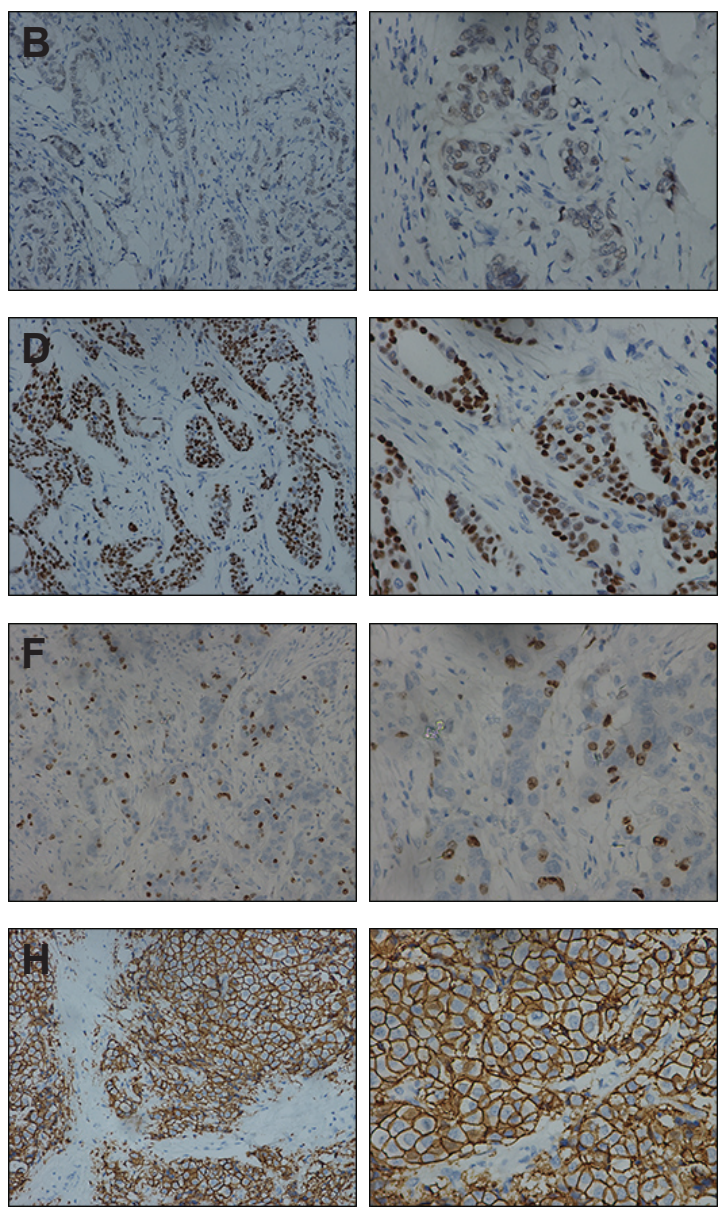

Figure I IHC of biomarkers in breast cancer.

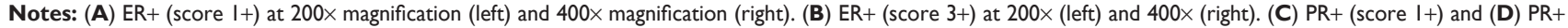
(score 3+). (E) Ki67 index was $<5 \%$ at $200 \times$ (left) and $400 \times$ (right). (F) Ki67 index was $30 \%$ at $200 \times$ (left) and $400 \times$ (right). (G) HER2 sample showing membranous staining (score $2+$ ) and $(\mathbf{H})($ score $3+)$.

Abbreviations: ER, estrogen receptor; HER2, human epidermal growth hormone receptor 2; PR, progesterone receptor.

\section{Statistical methods}

Experimental data were analyzed by SPSS 22.0 statistical software. Paired $t$-test for quantitative data and nonparametric Wilcoxon signed-rank test for ordinal categorical variables were used in the intra-group pairwise comparison. Test criterion a is $=0.05$. The consistency of ER, PR and HER2 status was analyzed by kappa analysis, with kappa value $>0.4$ regarded as good agreement and kappa value $>0.75$ considered as highly consistent. Mann-Whitney nonparametric test and independent $t$-test were used for performing intergroup comparisons between NAC group and control group. Univariate analysis of variance (ANOVA) and Pearson's correlation analysis for quantitative data, and Mann-Whitney nonparametric test and Spearman's correlation analysis for ordinal variables were applied to explore the relationship between biomarkers and potential influencing factors including age, menopausal status, tumor size, axillary lymph node status, ER, PR, HER2, molecular subtype, NAC regimen and STI. Multivariate ANOVA and regression analysis were then done to find the association and interaction between the biomarkers and these factors.

\section{Ethical approval}

This study was approved by the Ethics Committee of Sir Run Run Shaw Hospital (Approval No 20160705-1). Ethical wise, patients' consent is usually not required for data collection and research purposes if any step involved in collecting patients' information or research does not compromise with the quality of treatment and confidentiality of the patient. Throughout this research, patients' confidentiality was maintained at the strictest level.

\section{Results}

\section{Ki67 expression increased after core biopsy in the control group}

Ki67 expression value was much higher in SRS compared with core biopsy samples on using two paired-samples $t$-test, with mean values of $24 \%$ and $19.1 \%$, respectively $(P<0.01)$. 
Table 2 Quantitative changes in ER, PR and HER2 expression between paired CNB and SRS in the control group

\begin{tabular}{llll}
\hline Number & ER & PR & HER2 \\
\hline Total number of patients & I23 & 123 & 123 \\
Increase in cases (\%) & I I (8.9) & $27(22)$ & $8(6.5)$ \\
Decrease in cases (\%) & $23(18.7)$ & $17(13.8)$ & $7(5.7)$ \\
Negative $\rightarrow$ positive (\%) & $3(2.4)$ & $13(10.1)$ & $0(0)$ \\
Positive $\rightarrow$ negative (\%) & $7(6)$ & $7(5.7)$ & $2(1.6)$ \\
Concordance rate (\%) & 72.30 & 64.20 & 87.80 \\
Kappa value & 0.62 & 0.5 & 0.78 \\
P-value & 0.069 & 0.194 & 0.747 \\
\hline
\end{tabular}

Abbreviations: CNB, core needle biopsy; ER, estrogen receptor; HER2, human epidermal growth hormone receptor 2; PR, progesterone receptor; SRS, surgical excision samples.

No significant difference was found in the expression of ER, PR and HER 2 between CNB and SRS by Wilcoxon signedrank test ( $P=0.58,0.06$ and 0.67 , respectively), as shown in Table 2. Additionally, correspondence analysis showed good overall agreement in ER, PR and HER2 expression in both samples with concordance rates of $72.3 \%, 64.2 \%$ and $87.8 \%$ and kappa values of $0.62,0.5$ and 0.78 , respectively.

\section{Ki67 and PR expressions decreased after NAC}

In the NAC group, statistically significant difference in PR and Ki67 expression was observed between biopsy sample and post-NAC surgical excision $(P=0.009$ and 0.02 , respectively). Ki67 value decreased after NAC, with the mean value decreasing from $28.6 \%$ to $22.9 \%(P=0.02$; Tables 3 and 4$)$. Due to the same median PR value before and after NAC, mean PR value was used for comparing as an alternative. A reduction in mean PR score from 0.98 to 0.78 after NAC was observed in the NAC group ( $P=0.009$; Table 4). In addition, consistency analysis showed a relatively lower kappa value of 0.5 and a concordance rate of $64.2 \%$ in PR expression than those in ER, PR and HER2 expressions. No significant difference was found in ER and HER2 expression

Table 3 Quantitative changes in ER, PR and HER2 expression between paired CNB and SRS in NAC group

\begin{tabular}{llll}
\hline Number & ER (NAC) & PR (NAC) & HER2 (NAC) \\
\hline Total number of patients & 138 & 138 & 142 \\
Increase in cases (\%) & $10(7.2)$ & $13(9.4)$ & $20(14)$ \\
Decrease in cases (\%) & $24(17.4)$ & $28(20.2)$ & $20(14)$ \\
Negative $\rightarrow$ positive (\%) & $6(4.3)$ & $10(7.2)$ & $6(4.2)$ \\
Positive $\rightarrow$ negative (\%) & $7(5.1)$ & $16(11.6)$ & $5(3.5)$ \\
Concordance rate (\%) & 75.40 & 70 & 71.80 \\
Kappa value & 0.605 & 0.478 & 0.611 \\
P-value & 0.158 & 0.009 & 0.707 \\
\hline
\end{tabular}

Abbreviations: CNB, core needle biopsy; ER, estrogen receptor; HER2, human epidermal growth hormone receptor 2; NAC, neoadjuvant chemotherapy; PR, progesterone receptor; SRS, surgical excision samples.
Table 4 Expression changes in Ki67 and PR between paired CNB and SRS

\begin{tabular}{lllll}
\hline Group & $\begin{array}{l}\text { Total } \\
\text { number }\end{array}$ & $\begin{array}{l}\text { Pre-Ki67 } \\
\text { level (mean) }\end{array}$ & $\begin{array}{l}\text { Post-Ki67 } \\
\text { level (mean) }\end{array}$ & P-value \\
\hline Control group & 123 & $19.10 \%$ & $24.40 \%$ & $<0.01$ \\
NAC group & 86 & $28.60 \%$ & $22.90 \%$ & 0.02 \\
\hline & Total & Pre-PR level & Post-PR & P-value \\
& number & (mean) & level (mean) & \\
\hline Control group & 123 & 1.26 & 1.38 & 0.194 \\
NAC group & 138 & 0.98 & 0.78 & 0.009 \\
\hline
\end{tabular}

Abbreviations: CNB, core needle biopsy; NAC, neoadjuvant chemotherapy; PR, progesterone receptor; SRS, surgical excision samples.

after NAC. The concordance rates of ER and HER2 were $75.4 \%$ and $71.8 \%$ with kappa values of 0.605 and 0.611 , respectively, which is also regarded as a good agreement.

\section{Comparison on marker conversion between control and NAC groups}

We observed contradictory results for changes in the levels of Ki67, with an increase observed in the control group and a decrease in the NAC group. Based on intergroup comparison by independent $t$-test, this difference was statistically significant $(P \leq 0.01)$. Regarding changes in PR levels, with a decrease in the NAC group and no change in the control group, Mann-Whitney nonparametric test confirmed a significant PR decrease in the NAC group $(P=0.003)$. No significant difference was found in biomarker changes for $\mathrm{ER}$ and HER 2 between the control and NAC groups $(P=0.962$ and 0.86 , respectively).

Therefore, taken together, core sample is more accurate for evaluating Ki67 expression compared with subsequent surgical excisions. The increase in Ki67 value after CNB could lead to confusion in the molecular subtype determination at surgery in breast cancer. NAC exerted a genuine effect on PR and Ki67 alterations. Repeat testing of molecular biomarkers following neoadjuvant treatment is recommended for learning residual disease and managing subsequent adjuvant chemotherapy.

\section{Factors associated with $\mathrm{Ki} 67$ increase after CNB in the control group}

We further investigated potential factors associated with Ki67 increase by using univariate ANOVA and Pearson's correlation analysis in the control group (Table 5). The statuses of pathologic lymph node, PR and HER2 were found to be significantly associated with Ki67 change in surgical excisions after $\mathrm{CNB}$, while other factors including molecular subtype and STI had no influence. 
Table 5 Univariate analysis of potential clinicopathologic factors associated with $\mathrm{Ki} 67$ increase after CNB in the control group

\begin{tabular}{ll}
\hline Clinicopathologic factors & $P$-value \\
\hline Age & 0.496 \\
Menopause status & 0.134 \\
Pathologic tumor size & 0.603 \\
Pathologic lymph node status & 0.014 \\
Estrogen receptor & 0.053 \\
Progesterone receptor & 0.049 \\
HER2 & 0.005 \\
Molecular subtype & 0.49 \\
Surgery time interval & 0.059 \\
\hline
\end{tabular}

Abbreviations: CNB, core needle biopsy; HER2, human epidermal growth hormone receptor 2 .

Subgroup analysis showed that patients with axillary lymph node involvement had a higher Ki67 increase than those without, with the mean increase being $8.3 \%$ and $3.18 \%$, respectively ( $P=0.016$; Table 6$)$. Besides, patients with PR- or HER2+ showed more obvious Ki67 elevation after biopsy ( $P=0.049$ and 0.005 , respectively). However, multivariate ANOVA only demonstrated the significance of lymph node status and the interaction effect of PR and HER 2 status with $P$-values of 0.008 and 0.028 , respectively, which means the effects of PR and HER2 on Ki67 change are exerted by the combination of these two proteins rather than an individual factor.

\section{Factors associated with $\mathrm{Ki} 67$ and PR decrease after NAC}

Based on Mann-Whitney nonparametric test and Spearman's correlation analysis, menopause status and molecular subtype were found to be significantly correlated with the decrease in PR expression with $P$-values of 0.006 and 0.002 , respectively (Table 7). Further subgroup analysis showed that PR decrease was more obvious in premenopausal patients and

Table 6 Subgroup analysis of Ki67 increase after CNB in the control group

\begin{tabular}{|c|c|c|c|c|c|}
\hline Subgroup & Number & $\begin{array}{l}\text { Mean } \\
\text { Ki67\% } \\
(\mathrm{CNB})\end{array}$ & $\begin{array}{l}\text { Mean } \\
\text { Ki67\% } \\
\text { (SRS) }\end{array}$ & $\begin{array}{l}\text { Mean Ki67\% } \\
\text { change } \\
\text { (SRS-CNB) }\end{array}$ & $\overline{P \text {-value }}$ \\
\hline All populations & 123 & 19.10 & 24.40 & 5.30 & $<0.01$ \\
\hline Lymph node- & 66 & |8.7| & 21.89 & 3.18 & 0.016 \\
\hline Lymph node+ & 55 & 19.55 & 27.79 & 8.30 & \\
\hline PR- & 50 & 20.20 & 26.20 & 6.00 & 0.049 \\
\hline PR+ & 73 & 17.95 & 22.92 & 4.90 & \\
\hline HER2- & 98 & 15.20 & 20.54 & 5.29 & 0.005 \\
\hline HER 2+ & 25 & 33.20 & 38.89 & 5.60 & \\
\hline
\end{tabular}

Abbreviations: CNB, core needle biopsy; HER2, human epidermal growth hormone receptor 2; PR, progesterone receptor; SRS, surgical excision samples.
Table 7 Univariate analysis of potential clinicopathologic factors associated with PR and Ki67 decreases after NAC in the NAC group

\begin{tabular}{lll}
\hline Clinicopathologic factors & $P$-value (PR) & $P$-value (Ki67) \\
\hline Age & 0.182 & 0.559 \\
Menopausal status & 0.006 & 0.581 \\
Tumor size by ultrasound & 0.19 & 0.759 \\
Clinical lymph node status & 0.65 & 0.305 \\
Estrogen receptor & 0.0845 & 0.42 \\
HER2 & 0.416 & 0.958 \\
Molecular subtype & 0.002 & 0.08 \\
NAC regimen & 0.318 & 0.668
\end{tabular}

Abbreviations: HER2, human epidermal growth hormone receptor 2; NAC, neoadjuvant chemotherapy; PR, progesterone receptor.

Luminal A breast cancer. Due to the same median PR value pre- and post-NAC in each subgroup, mean PR was used as an alternative. A slight increase in post-NAC PR value by 0.04 was observed in postmenopausal patients, while a significant decrease by 0.46 was found in premenopausal patients. In addition, PR expression change showed different trends in molecular subtypes: a decrease in Luminal A and Luminal B, a slight increase in HER2+ and no change in triple negative. PR decrease was more obvious in Luminal A group than in Luminal B ( $P=0.002$; Table 8$)$. Regression analysis had not been done due to the limited number of patients.

No potential clinicopathologic feature was found to be significantly correlated with the decrease in Ki67 expression after NAC by univariate ANOVA, possibly due to the limited number of patients with fully recorded Ki67 value; only 86 patients in the NAC group had recorded paired Ki67 value.

\section{Discussion}

In this study, we reported the significant changes in receptor expression following core biopsy and subsequent NAC, and its associated influencing factors. Ki67 value was significantly increased in surgical excisions after initial core biopsy,

Table 8 Changes in PR values after NAC in different subgroups

\begin{tabular}{|c|c|c|c|c|c|}
\hline Subgroup & Number & $\begin{array}{l}\text { Mean PR } \\
\text { (CNB) }\end{array}$ & $\begin{array}{l}\text { Mean PR } \\
\text { (SRS) }\end{array}$ & $\begin{array}{l}\text { Mean PR } \\
\text { change } \\
\text { (SRS-CNB) }\end{array}$ & $P$-value \\
\hline All populations & 138 & 0.98 & 0.78 & -0.2 & 0.009 \\
\hline Premenopause & 69 & 1.23 & 0.77 & -0.46 & 0.006 \\
\hline Postmenopause & 65 & 0.77 & 0.81 & 0.04 & \\
\hline Luminal A & 14 & 2.43 & 1.71 & -0.72 & 0.002 \\
\hline Luminal B & 49 & 1.22 & 0.9 & -0.32 & \\
\hline HER 2+ & 27 & 0.07 & 0.11 & 0.04 & \\
\hline $\mathrm{TN}$ & 29 & 0 & 0 & 0 & \\
\hline
\end{tabular}

Abbreviations: CNB, core needle biopsy; HER2, human epidermal growth hormone receptor 2; NAC, neoadjuvant chemotherapy; PR, progesterone receptor; SRS, surgical excision sample; $\mathrm{TN}$, triple negative. 
which was related with axillary lymph node status and the interaction of PR and HER2 status. A significant decrease in the expression of PR and Ki67 after NAC was observed. Further stratified analysis showed PR decrease was more obvious in premenopausal and Luminal A patients.

Several studies have reported only fair to moderate agreement for Ki67 testing between core samples and surgical excisions. ${ }^{16,17,19}$ Recent studies by Chen et $\mathrm{al}^{16}$ and Kim et al ${ }^{17}$ showed an increase in Ki67 value in SRS and identified the associated risk factors. These results are concordant with our study showing that Ki67 value was higher at surgery than at biopsy $(24.4 \%$ vs $19.1 \%, P<0.01)$, while ER, PR and HER2 statuses were consistent in both samples. Previous authors suggested that HER 2 overexpression probably played a role in postsurgery stimulation of growth of breast carcinoma cells. Tagliabue et al demonstrated that residual breast carcinomas that had been surgically removed showed a significant increase in proliferation and wound drainage fluid and postsurgical serum samples from patient stimulated in vitro growth of HER2-overexpressing breast carcinoma cells. ${ }^{20}$ Thus, compared with surgical excisions, core samples were more accurate in evaluating breast cancer biomarker status and molecular subtype.

We further analyzed potential clinicopathologic factors associated with Ki67 increase after biopsy and found that lymph node status was an independent influencing factor $(P=0.008)$. Patients with lymph node involvement had a more obvious increase in surgical samples, indicating that $\mathrm{Ki} 67$ increase was associated with a worse disease outcome. Tumors with invasive behavior showed a higher level of cell proliferation in wound healing process, characterized by a more obvious Ki67 increase. Thus, for better management of aggressive tumor, it is suggested to shorten the time interval between biopsy and surgery. Additionally, a case-control study consisting of 416 breast cancers and 1,156 healthy women in China found that breast tumor biopsy, albeit confirmed benign pathologically, was an independent risk factor of breast cancer. ${ }^{21}$ This proposed the assumption that cell proliferation in the wound healing process after CNB could also lead to breast cancer.

We also found on univariate analysis that patients with PR- or HER2+ status showed more obvious Ki67 elevation after biopsy ( $P=0.049$ and 0.005 , respectively). However, multivariate ANOVA only demonstrated the interaction effect of PR and HER2 status with a $P$-value of 0.028 , which means the effect of PR and HER2 on Ki67 change is exerted by the combination of these two proteins rather than an individual one.
Although NAC has been well demonstrated to change ER, PR, HER2 and Ki67 expressions in breast cancer, controversial results were found on the pattern of biomarker changes. Several preanalytical and analytical factors (such as variation in fixation, quality of staining and observer variation) can result in discrepancies in biomarker status. After demonstrating a CNB-associated Ki67 increase, our study included a control group of paired tumor samples from the same study period and undergoing the same fixation, processing and staining protocols. The small extent of marker change within this control group supports that the changes identified in the test group are due to genuine treatment effect.

By this method, decreases in PR and Ki67 expression following NAC were observed in the case group $(P=0.003$ and $P<0.01$, respectively), while no statistically significant difference in ER and HER2 status was found between cases and controls. This is supported by a recent study conducted by Gahlaut et al including 243 cases and 113 controls, which reported that PR status changed significantly after NAC. Thirteen cases changed status from PR positive to negative and only five from negative to positive. ${ }^{22}$ Possible mechanisms of molecular alteration include selection of resistant tumor cells to NAC left in the residual disease with sensitive cells being targeted and the survival mechanism of tumor cells by changing the cellular molecular pathway leading to resistance to a specific therapy. ${ }^{13}$ In clinical practice, a change in HR status will bring adjustment of treatment strategy. Patients with a switch from ER/PR positive to negative will be less benefited from endocrine therapy. Thus, based on our findings, we recommend repeat testing of ER, PR, Ki67 and HER2 following neoadjuvant treatment for better disease management.

Further stratified analysis showed that menopause status and molecular subtype were two factors associated with a reduction of $\mathrm{PR}$ expression. $\mathrm{PR}$ decrease was more obvious in premenopausal and Luminal A patients $(P=0.006$ and 0.002 , respectively), which was supported by Enomoto's study showing a significant decrease of PR level in premenopausal breast cancer patients. ${ }^{10}$ Luminal A breast cancer subtype has the most favorable prognosis in all breast cancer subtypes. ${ }^{23,24}$ Taken together, PR decrease after NAC seemed to relate with a favorable patient outcome. This hypothesis was supported by Montagna et al's study, a long-term follow-up of 904 breast cancer patients, which showed that the decrease in PR and Ki67 expression after NAC correlated with improved outcome in terms of DFS, and that the decrease of Ki67 expression to $<20 \%$ of the cells after NAC was associated with better outcome in terms of DFS and overall survival. ${ }^{11}$ 
For Ki67, several studies have demonstrated that the decrease in posttreatment Ki67 level is a positive predictor for patient outcome. ${ }^{11,25}$ However, no significant factor was identified to correlate with this decrease in our study, possibly due to the limited number of patient samples.

To the best of our knowledge, this is the most comprehensive study to analyze the effect of NAC on the change of ER, PR, HER2 and Ki67 after reporting CNB-associated Ki67 increase in the same study. We also suggest this method in future neoadjuvant breast cancer studies to minimize the interference of CNB and other confounding factors. Neoadjuvant breast cancer studies, utilizing the change of presurgical Ki67 expression to evaluate tumor cell proliferation and the efficacy of neoadjuvant therapy, have to take CNB-associated Ki67 increase into consideration.

There were several limitations in our study. First, the number of enrolled patients was relatively unsatisfactory, and missing data of some cases resulted in an even smaller sample size. Second, ER and PR data of almost half of the patients were recorded as ranked scores instead of percentage, leading to declined power of statistical test. The absence of fluorescence in situ hybridization assessment for HER2 might cause false-negative cases. Third, several confounding factors (such as variation in fixation, quality of staining and observer variation) can result in discrepancies in biomarker status. Our study included a control group of tumor samples from the same study period and undergoing the same fixation, processing and staining protocols, in order to demonstrate genuine treatment effect. In spite of these efforts, such analytical factors are unavoidable.

\section{Conclusion}

Our study has demonstrated an elevated level of Ki67 in surgical excisions caused by the stimulation of CNB. Compared with subsequent surgical samples, core samples could provide more reliable information on molecular subtype status. For aggressive tumor not requiring NAC, it is suggested to shorten the time interval between biopsy and surgery. PR and Ki67 expression decreased following NAC, which could be used as potential prognostic factors for better outcome. We recommend repeat testing of ER, PR, Ki67 and HER2 following neoadjuvant treatment for the sake of better disease management. We also suggest this method in future neoadjuvant breast cancer studies to minimize the interference of CNB and other confounding factors.

\section{Acknowledgments}

The authors are grateful to Yanyan-Chai for her excellent data management and the statistical team in Sir Run Run hospital for its kindly help. We would like to acknowledge the helpful comments on this paper received from our reviewers and editors. This work was supported by a grant from the National Key Basic Research Program of China (973 Program; 2014CB744505).

\section{Disclosure}

The authors report no conflicts of interest in this work.

\section{References}

1. Mauri D, Pavlidis N, Ioannidis JP. Neoadjuvant versus adjuvant systemic treatment in breast cancer: a meta-analysis. J Natl Cancer Inst. 2005; 97(3):188-194.

2. Abrial SC, Penault-Llorca F, Delva R, et al. High prognostic significance of residual disease after neoadjuvant chemotherapy: a retrospective study in 710 patients with operable breast cancer. Breast Cancer Res Treat. 2005;94(3):255-263.

3. Leone JP, Leone J, Vallejo CT, et al. Sixteen years follow-up results of a randomized phase II trial of neoadjuvant fluorouracil, doxorubicin, and cyclophosphamide (FAC) compared with cyclophosphamide, methotrexate, and 5-fluorouracil (CMF) in stage III breast cancer: GOCS experience. Breast Cancer Res Treat. 2014;143(2):313-323.

4. Romero A, Garcia-Saenz JA, Fuentes-Ferrer M, et al. Correlation between response to neoadjuvant chemotherapy and survival in locally advanced breast cancer patients. Ann Oncol. 2013;24(3):655-661.

5. Symmans WF, Peintinger F, Hatzis C, et al. Measurement of residual breast cancer burden to predict survival after neoadjuvant chemotherapy. J Clin Oncol. 2007;25(28):4414-4422.

6. Chavez-MacGregor M, Gonzalez-Angulo AM. Breast cancer, neoadjuvant chemotherapy and residual disease. Clin Transl Oncol. 2010; 12(7):461-467.

7. Ge WK, Yang B, Zuo WS, et al. Evaluation of hormone receptor, human epidermal growth factor receptor-2 and Ki-67 with core needle biopsy and neoadjuvant chemotherapy effects in breast cancer patients. Thorac Cancer. 2015;6(1):64-69.

8. Zhou X, Zhang J, Yun H, et al. Alterations of biomarker profiles after neoadjuvant chemotherapy in breast cancer: tumor heterogeneity should be taken into consideration. Oncotarget. 2015;6(34):36894-36902.

9. Jin X, Jiang YZ, Chen S, Yu KD, Shao ZM, Di GH. Prognostic value of receptor conversion after neoadjuvant chemotherapy in breast cancer patients: a prospective observational study. Oncotarget. 2015;6(11): 9600-9611.

10. Enomoto Y, Morimoto T, Nishimukai A, et al. Impact of biomarker changes during neoadjuvant chemotherapy for clinical response in patients with residual breast cancers. Int J Clin Oncol. 2016;21(2): 254-261.

11. Montagna E, Bagnardi V, Viale G, et al. Changes in PgR and Ki-67 in residual tumour and outcome of breast cancer patients treated with neoadjuvant chemotherapy. Ann Oncol. 2015;26(2):307-313.

12. Miglietta L, Morabito F, Provinciali N, et al. A prognostic model based on combining estrogen receptor expression and Ki-67 value after neoadjuvant chemotherapy predicts clinical outcome in locally advanced breast cancer: extension and analysis of a previously reported cohort of patients. Eur J Surg Oncol. 2013;39(10):1046-1052.

13. van de Ven S, Smit VT, Dekker TJ, Nortier JW, Kroep JR. Discordances in ER, PR and HER2 receptors after neoadjuvant chemotherapy in breast cancer. Cancer Treat Rev. 2011;37(6):422-430.

14. Chen X, Yuan Y, Gu Z, Shen K. Accuracy of estrogen receptor, progesterone receptor, and HER2 status between core needle and open excision biopsy in breast cancer: a meta-analysis. Breast Cancer Res Treat. 2012;134(3):957-967.

15. Tagliabue E, Agresti R, Carcangiu ML, et al. Role of HER2 in woundinduced breast carcinoma proliferation. Lancet. 2003;362(9383): $527-533$. 
16. Chen X, Zhu S, Fei X, et al. Surgery time interval and molecular subtype may influence Ki67 change after core needle biopsy in breast cancer patients. BMC Cancer. 2015;15:822.

17. Kim HS, Park S, Koo JS, et al. Risk factors associated with discordant Ki-67 levels between preoperative biopsy and postoperative surgical specimens in breast cancers. PLoS One. 2016;11(3):e0151054.

18. Goldhirsch A, Wood WC, Coates AS, Gelber RD, Thurlimann B, Senn HJ; Panel members. Strategies for subtypes dealing with the diversity of breast cancer: highlights of the St. Gallen international expert consensus on the primary therapy of early breast cancer 2011. Ann Oncol. 2011;22(8):1736-1747.

19. Greer LT, Rosman M, Mylander WC, et al. Does breast tumor heterogeneity necessitate further immunohistochemical staining on surgical specimens? J Am Coll Surg. 2013;216(2):239-251.

20. Tagliabue E, Agresti R, Carcangiu ML, et al. Role of HER2 in woundinduced breast carcinoma proliferation. Lancet. 2003;362(9383): 527-533.
21. Xu YL, Sun Q, Shan GL, et al. A case-control study on risk factors of breast cancer in China. Arch Med Sci. 2012;8(2):303-309.

22. Gahlaut R, Bennett A, Fatayer H, et al. Effect of neoadjuvant chemotherapy on breast cancer phenotype, ER/PR and HER2 expression Implications for the practising oncologist. Eur J Cancer. 2016;60:40-48.

23. Mahmood H, Faheem M, Mahmood S, Sadiq M, Irfan J. Impact of age, tumor size, lymph node metastasis, stage, receptor status and menopausal status on overall survival of breast cancer patients in Pakistan. Asian Pac J Cancer Prev. 2015;16(3):1019-1024.

24. Lyman GH, Kuderer NM, Lyman SL, et al. Menopausal status and the impact of early recurrence on breast cancer survival. Cancer Control. 1997;4(4):335-341.

25. Jones RL, Salter J, A'Hern R, et al. The prognostic significance of Ki67 before and after neoadjuvant chemotherapy in breast cancer. Breast Cancer Res Treat. 2009;116(1):53-68.

\section{Publish your work in this journal}

OncoTargets and Therapy is an international, peer-reviewed, open access journal focusing on the pathological basis of all cancers, potential targets for therapy and treatment protocols employed to improve the management of cancer patients. The journal also focuses on the impact of management programs and new therapeutic agents and protocols on

\section{Dovepress}

patient perspectives such as quality of life, adherence and satisfaction. The manuscript management system is completely online and includes a very quick and fair peer-review system, which is all easy to use. Visit http://www.dovepress.com/testimonials.php to read real quotes from published authors. 